\title{
Simulation Tools and Methods of Physical Phenomena for Smart City
}

\author{
Eloi B. Keita*, Valery Monthe, Pierre-Yves Lucas and Bernard Pottier \\ University of Western Brittany, Lab-STICC - UMR/CNRS 6285, Brest, France \\ ${ }^{*}$ Corresponding author
}

\begin{abstract}
Many physical phenomena can be modeled as the propagation of sound or radio waves, the fluids flow, the distribution of gas, the dispersion produced in a river, etc. With the help of the AC we can deduce very realistic situations. In previous work, we simulated the propagation of sound in the city using cellular automata, taking into account the urban parameters. In that works, we used horizontal bars to simulate the presence of obstacles (buildings and others) in the sound propagation environment. These obstacles have been defined with arbitrarily fixed coefficients of resistance. In this paper, we rely on the models and algorithms proposed in previous works, to observe the behavior of our simulations of sound, in a concrete environment of obstacle. For this we use NetGen[1], which is a map processing tool. PickCell allows us to analyze the behavior of sound in a concrete environment
\end{abstract}

Keywords-Wireless sensor networks, cellular automata;sound propagation simulation; graphics accelerators

\section{INTRODUCTION}

Physical world and information systems unification is a major achievement during this last decade. Communication systems, especially wireless technology, and research in physical sensors field have taken a major part in this integration.

The interface between the physical word and a computer can be used in two ways. First, from a local perspective, where such a system can control for instance a single object or provide a personal assistance. Moreover, in a distributed system, this interface collects and analyze sparse data to make decisions, for instance about resource savings: to turn off streetlights, to guide to a parking spot. Wireless sensor networks are part of this second category. Thus, this paper is focused on sound propagation simulation[2] in urban environment, in a distributed manner.

The key point of this work is a cellular representation of the city in order to model streets, gardens, ring roads, buildings, and rivers. Geo-referenced image analysis, complemented by database consultation, for example to retrieve elevation data, generates this cellular model. Then, this model is converted into a system made of interconnected processes, that can re-produce many collective behaviors, whether physical or digital. We have developed a cellular automata that models sound propagation, including reflection and refraction, working on a graphics accelerator. Finally, we have produced a coupling method between observation systems by sensor networks and physical systems.

\section{CELlular SySTEMS AND ENVIRONMENT}

\section{A. Synthesis Parameters, Evaluation of Cellular Systems}

In this section, we study the methods to configure cell synthesis and the impact of this configuration on the resulting sim-ulators. This study is important in regards to both the quality of the discrimination between physical areas and calculation load resulting. The synthesis of network is being performed, it is possible to provide static characteristics (section III) and use simulator of characteristics measured dynamically (section IV-A). The achievement result from these assessments that have been run on several cellular network[3] resume below:

- nigerCarte: the maps(2a) show simplified information such as roads, buildings or blocks, names, green areas and wetlands.

- nigerPhoto: aerial photography (Niger River, figure 2b).

- campusbatiments: limits of buildings on campus

- campusJardin: campus free zone both connectivity allow to present cellular zone obtained for the buildings firstly, paths, gardens or roads secondly.

Dynamic evaluations are focused on these four networks built from four neighborhoods: VonNeumann1 and VonNeumann2, Moore1 and Moore2. Section IV-A describes the algorithms compiled and evaluated for this test.

- Build and make: in this case regarding some representa-tive samples, it was necessary to demonstrate the feasibil-ity, the complexity and the performance of reproducible simulations on large-scale[?]. For this purpose, we have designed a tool to execute and generate automatically for displaying data from topological characterization.

- FanOut: number of output channels of a node

- MaxFanOut: maximum number of outbound channels from the same node into the network.

- MinFanOut: minimum number of outgoing channels from the same node into the network.

- Fannin: number of input channels of a node.

- Leader: Network identifier

- Diameter: number of steps to propagate the information into cellular system.

- Process: an AC cell - each process is a cell.

- Channels: communication links.

To summarize, the parameters of an incident are: 
- (SC) cellular system synthesized (there are 4 during this study: nigerCarte, nigerPhoto, campusBatiment, campusJardin)

- (CC) cellular connectivity, there are also 4 in this study (VN1, VN2, Moore 1, Moore 2),

- $(\mathrm{AD})$ distributed algorithm (in the case, we have 44 architectures by 5 distributed algorithms, which are 80 programs to simulate automatically)

The segmentation steers to draw a two-dimensional[4] table according to the selected sizes. The elements correspond to cells of system which are constituted at the beginning by small square and rectangular images. An example of that segmentation is described by figure $4 \mathrm{~b}$.

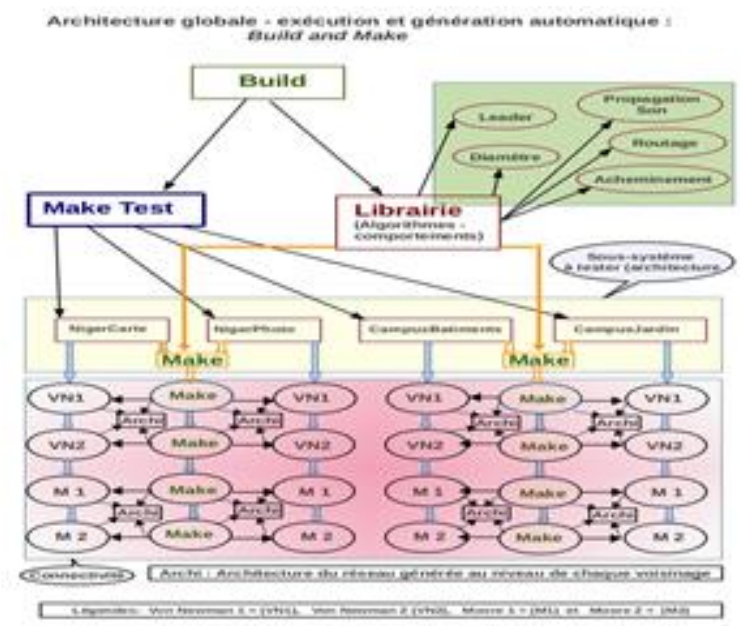

FIGURE I: SYNOPTIC OF AUTOMATIC SYSTEM OF GENERATION:

- 4 subsystems to be tested are nigerCarte, nigerPhoto, campusBatiment, campusJardin,

- 5 algorithmic functions are: leader, diameter, routing, transport, sound propagation,

- 4 cellular connectivity are: VN1, VN2, Moore1(M1) and Moore2(M2),

- 16 architectures are indicated per blocks "ARCHI", they'll be associated with each function to be tested ( 80 assessments).

\section{B. Cellular System Discrimination}

Obtaining of cellular system simulable can be define in 3 steps:

- Segmentation and clustering of cells;

- Neighborhood choice and production of abstracted architecture;

- Behaviors definition for physical process checking.

At the end of those 3 steps, we have a program available to represent quantity of physical phenomenon such as propagation (sound, light, radio), spreading (pollutants, insects), clustering and aggregations (insects), etc. It's possible to provide data to sensor networks and calculate the dimensions of these networks considering physical phenomenon.

\section{Classification}

The physical data are represented with an image made up of pixels containing 3 components Red, Green, Blue (RGB). From a map or aerial photograph, it's become easy to have that image (see figure 2).

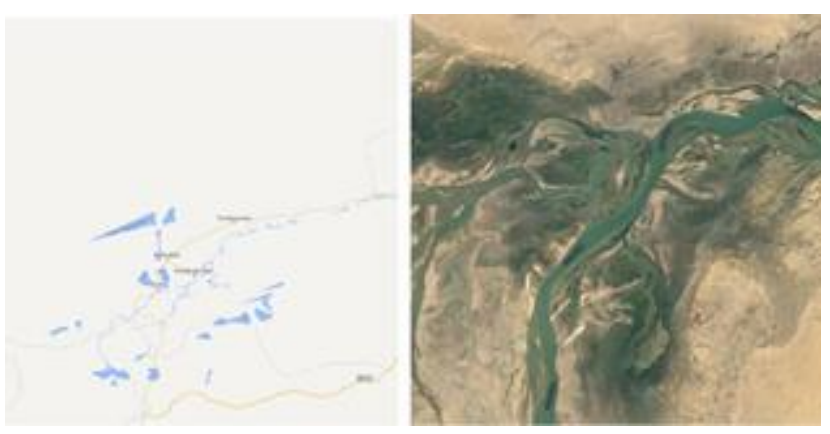

(a) Stream map in the region (b) Corresponding view from satellite

FIGURE II. HYDRO-GEOGRAPHY MAP IN A REGION OF AFRICA

Therefore we perform a systematic measure on all cells by calculating parameters such as the average of pixels on their three components (average(Red), average(Green), average(Blue)). its pixels.

Phase 1

For each cell of the grid

For each pixel inside a cell

For each color component from \{Red, Green, Blue\}

Update (min(color))

Update (max (color))

Update (total (color))

In order to proceed at the distribution per groups, we're also interested in general distribution of colors. It is necessary to have space taken by colors on all cells.

Phase 2:

For each cell of grid

For each component of color in (Red, Green, Blue

Update (minGlobal)

Update (maxGlobal)

Update (minMeanGlobal)

Update (maxMeanGlobal)

We have now for each parameter the val-

ues taken overall of images. For example

$\mathrm{I}=[\operatorname{minR} ; \operatorname{maxR}] ;[\min G ; \operatorname{maxG}] ;[\min B ; \operatorname{maxB}] . \quad$ Figure 3a

To see a statistical graph of colors on a photographic process tool.

Phase 3: 
For each interval, allocate a collection

For each cell, record into a collection

At the end of this operation the automatic groups are completed. PickCell tools provide possibility of manual combinations of classes: definition of a target class and ability to aggregate them to near classes (see the menu on figure III).
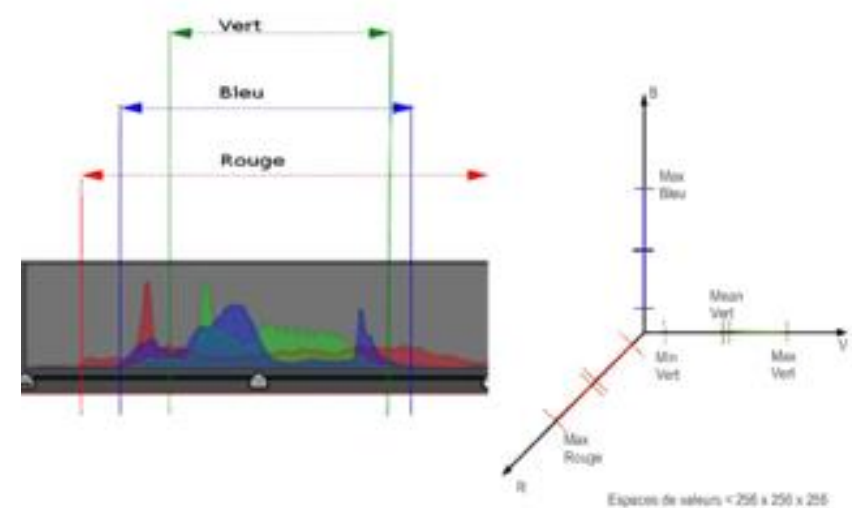

(a) Based on the pre-treatment re-sults shown in figure 6, the intervals [min, max] are shown for each color component (b) Classes and cells per color

FIGURE III: COLORS CLASSES

\section{Construction of classes}

It's possible now to use the parameters calculated in every cell. the goal is to get classes representing of cells affinities and the number of classes are depend on the number of divisions operated on interval I. A partition into two part will distribute the space of value into 23 sub-cubes and we can get then $8,27,64$ classes for $n=2 ; 3 ; 4$ partitions (see figure $3 b$ ).

\section{E. Segmentation and Classification Practice}

Figure $4 \mathrm{a}$ shows a view of the tool used to implement functions described in this document. The left column shows the classes generated from a satellite image pre-processed. The central image shows the left column selected class. we identify the criteria of classification: min, max, mean. At right in the bottom there is the choice of neighborhoods allowing the system process synthesis. The tool provides the current cellular system statistics. It also allows to set up classes combinations. This tool also allows to put in place sensors on the interesting zones and causing generation of abstract model for the sensor system. Therefore we have a direct interaction between the data manipulation and the planning of their observation. Figure 5a shows two views with the purpose to illustrate the qualities of this cell partitioning technique. Knowing that the tool can be improved, especially in its classification system.

\section{F. Pre-treatments}

Prior to process color with a standard treatment software which allows to highlight the interest zones (wetland namely in this simulation). This is a way for color configuration allowing to facilitate classes separation. The pre-treatment carried out with external tools can be useful in expectation of more sophisticated classification techniques to automatically perform discrimination.
In the figure $4 b$, the space is split into known size grid of cell. The size of this grid is $10 @ 10$ pixels.

\section{G. Generation of Network Process}

Figure 7 shows the practically of this generation with two choice for "Von Neumann" and "Moore". This generation is produced on an abstract model formed of connected process by link. This model is expressed on a small number of Smalltalk classes allowing management of some attributes such as location.

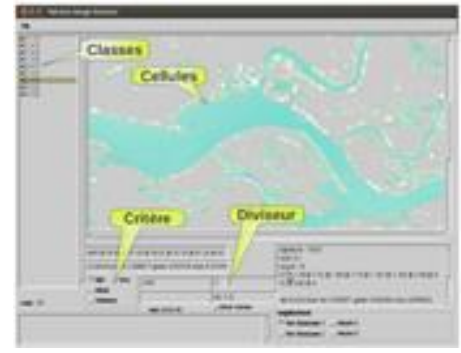

(a) Classes managment tool

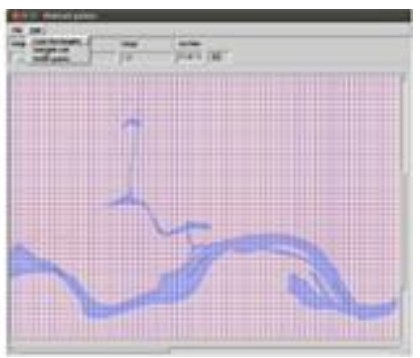

(b) Pickcell
FIGURE IV. SOMES USED TOOLS

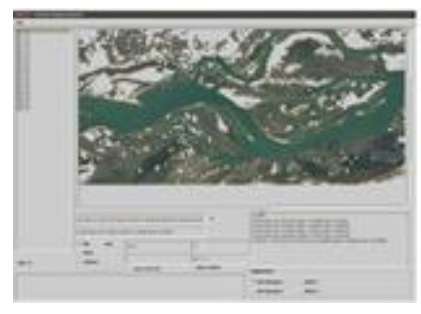

(a) On tombuctou map

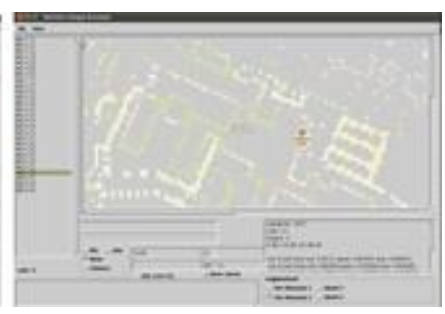

(b) On UBO campus map
FIGURE V. CELL PARTITIONING METHOD

Figure 7 shows a view of NetGen, control tool for code generation. This tool can support cellular systems and sensor of network by producing graphs, Occam[5] code or CUDA [6] code. It recognizes:

- $\quad$ localized processes, their fan-out, behavior procedure and geographical information,

- the parallel composition of these processes.

We obtain the generation of Occam code similar to those of NetGen sensor network by using the command window shown on Figure 7. The model displayed at the right on the window is similar to the extract seen below where we notice the horizontal and vertical neighbors of cellular process.

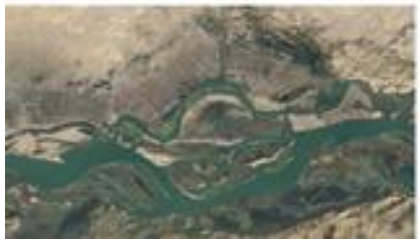

(a) Initial Image

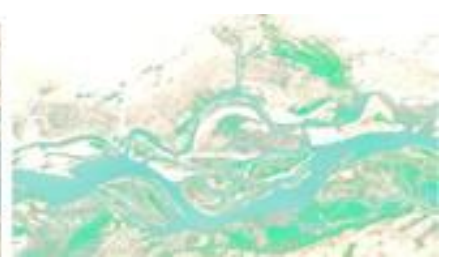

(b) Image processed and enhance-ment of color range

FIGURE VI: COLOR PROCESSING 


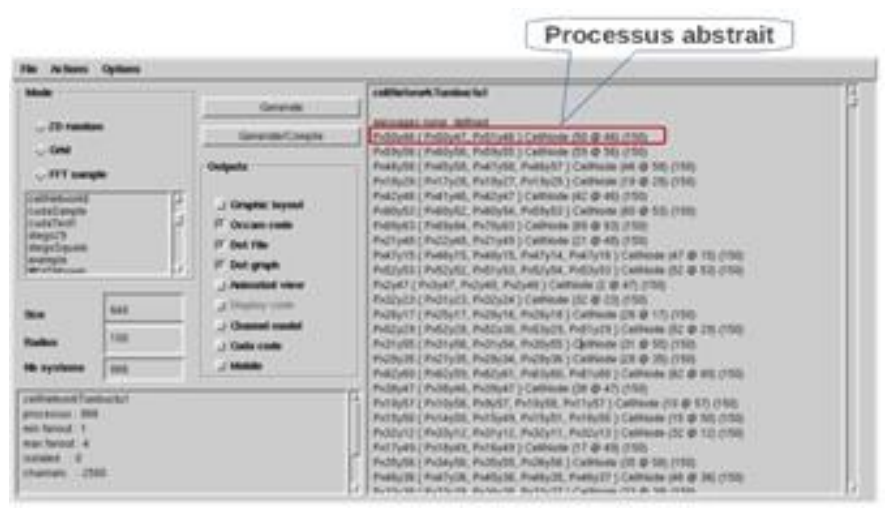

FIGURE VII: TEXTUAL PRESENTATION OF ABSTRACT MODEL FOR A TYPE OF CELLULAR SYSTEM SUCH AS VON NEUMANN DISTANCE

\section{Statistical StUdies IN THE City}

\section{A. Parameters}

In light of simulation of sound propagation, we interest to open space and buildings. The open spaces are zones where the soundwaves, radio signals or electromagnetic waves can be propagate in straight line. This postulate assume that obstacles are considered only things (buildings, walls, trees. . . ) whose the attenuation coefficients are greater than or equal to that from air. By applying "law of conservation of mass" which determine the isolation[7].

$$
\mathrm{R}=20 \times \log (w M 2 r c)
$$

with: $R$ : acoustic intensity level in decibel $(\mathrm{dB}) ; W$ : angular velocity (beat) in rad.s; $M$ : area density (kg.m-2); $Z$ : Acoustic impedance $410 \mathrm{~Pa} . \mathrm{s} / \mathrm{m} 3$; the "law of conservation of Mass" means that in the ideal case of an infinitely large wall (or highly absorbed), $\mathrm{R}$ increase by $6 \mathrm{~dB}$ per double frequency and by $6 \mathrm{~dB}$ per double of area density.

\section{B. Results of Quantitative Statistical Analysis}

In order to quantify the charge of calculations induced by different choice of parameters offered by PickCell, we discriminated few solid zones (buildings) and hollow (gardens).

\section{Groung Floor Description and Behavior}

There is no fundamental difference between physical cell behavior simulated and the synchronous sensor network simulated by NetGen [1]. Treatment programmed to these network are immediately transferable to cellular networks:

- distributed algorithms for networks data: leaders calcu-lations, diameters, routing and transports apply to cell assembly.

- $\quad$ physical models: cellular automaton purely local, distributed average, sound propagation, flow, gas, water, etc.
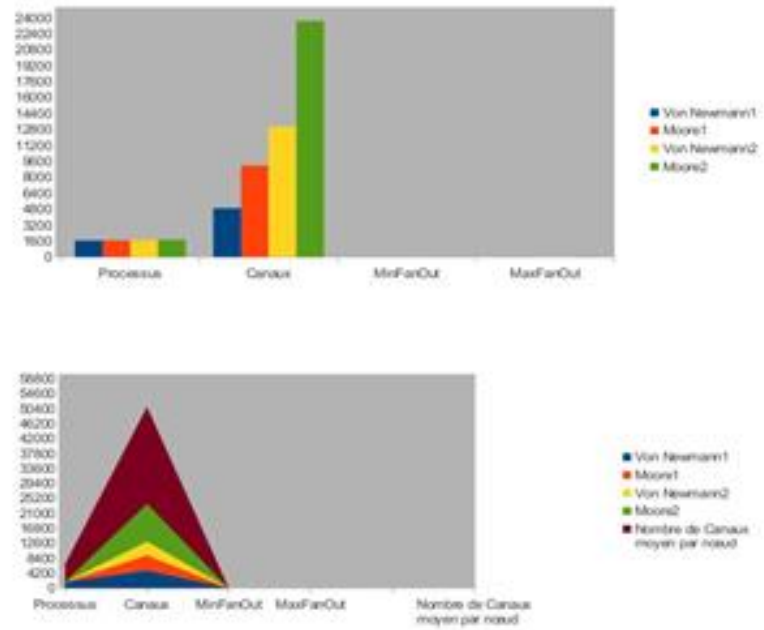

FIGURE VIII: BUILDINGS (OBSTACLE OF WAVES PROPAGATION) DIVIDER:4

\begin{tabular}{|l|l|}
\hline Leader & Max des ID \\
\hline Diametre & Max des distances $d_{i j}$ \\
\hline Routes & table des liens $\mathrm{i}->\mathrm{j}$ \\
\hline Acheminement & transport des paquets \\
\hline Position & repère xyz d'une cellule \\
\hline propagation & direction, diffusion, réflexion, ou absorption des ondes \\
\hline \multicolumn{2}{|c|}{ FIGURE IX. STATISTICAL STUDY ON THE NEIGHBORHOODS: } \\
VARIATION CC VS VARIATION SC
\end{tabular}

\section{Structures of Cellular Data}

Theses structures must be described by the programmer of cellular automaton and support the description of the local state and the evolution calculation: consistent progression with synchronous model, coded in PosDircellnode-include6: (state(t) inputs $=>$ state $(\mathrm{t}+1)$, outputs $)$.

\section{STATISTICAL STUDY EXAMPLE OF HYDRO-GEOGRAPHY IN TOMBOUCTOU REGION (MALI)}

Routing demonstration and packet transport alongside of Niger River (Djoliba) in the Tombouctou area in Mali. We can also calculated: the parameter of the leader, the diameter of the network, parameters of routing, transport of network (number of lost packet, collision detection, etc.)

\begin{tabular}{|l|l|l|l|l|}
\cline { 2 - 5 } \multicolumn{4}{c|}{} & \multicolumn{4}{c|}{ Analyse statistique } \\
\hline CC variable : VN1, VN2, MI et M2 - SC constant : nigerCarte - Granularité : $10 \Theta 10$ \\
\hline Voisinages Min & Processus & Canaux & MinFanOut & MaxFanOut \\
\hline Von NeumannI & 1653 cellules & 4900 & 1 & 4 \\
\hline Moore1 & 1680 cellules & 9190 & 1 & 8 \\
\hline Vos Neumann2 & 1701 cellules & 13078 & 1 & 12 \\
\hline Moore2 & 1735 cellules & 23700 & 1 & 24 \\
\hline
\end{tabular}

FIGURE X: THE COASTLINE OF NIGER RIVER AT TOMBOUCTOU (MALI) -

Divider: 4

FIGURE 11: Indicators of behavior tested. Some example of dynamic processing 


\section{E. Specification of Behaviors: Diameter and Distance of} Network Calculation

(Index of the shortest path from one cell to cell reachable, the long path inside a system) A full program of Occam is constituted of 3 files, $1^{\text {st }}$ and $3^{\text {rd }}$ are automatically generated:

1) architecture:concurrent cellular system reproducing image of synthesized class, conform with selected neighborhood.

2) behavior: behavior and notoriously procedures which describes the behavior and the state of the cell.

3) data: data from class described in Occam, based in an overall table, and read by each process as given parameter.

The algorithms used in this work are composed of basic distributed examples:

- the leader: network identifier allowing a mobil to obtain the identifier of the network met on his route,

- the diameter: defined as the maximum distance between any two nodes, allowing to recognize the number of rounds require propagation of information into network.

Two networks are also tested: transportation and routing. Finally, two algorithms for specific physical propagation are

also tested: position and direction, and propagation of sound-wave.

\section{CONCLUSION AND PERSPECTIVES}

The case study of the Niger River in the Timbuktu region, where four neighborhoods have been tested (figure 2) is based on these two approaches. Thus, for this study, a rigorous analysis was carried out between different neighborhoods and changes in classes. This allowed us to determine that this method actually provides new simulation solutions of physical phenomena. The compilation of over 3000 Occam processes is at the limit of the capacity of KROC compiler, while the same compilation is much faster ran on Cuda. As for this

Many physical phenomena can be modeled as the propagation of sound or radio waves, the fluids flow, the distribution of gas, the dispersion produced in a river, etc. with the help of

Difficulty of quantifying, the use of graphics accelerators can come in handy (Graphic Processing Units, GPU)[8] specially in massively parallel SIMD in run mode. The AC being a relatively new numerical and original method, it is important to situate their performance compared to conventional methods especially the differential equations[9].

The AC we can deduce very realistic situations. In this work, the prospects prove quite interesting. One can thus model the University of Western Brittany campus in 2 different ways:

- the propagation of sound waves in the "gardens" of the campus (without obstacles): detection, localization of sound sources at rallies, crowd movements, exceptional events on campus, etc.
- Sound propagation in and through buildings (obstacles): calculating the resistance of buildings to sound waves depending on the power of the sound source, location, provident noise[10], etc.

Finally, in addition to the technical advantages of AC over mathematical methods noted in this work, the use of the results is wide and in varied fields, such as:

- mobile: piloting a drone, a boat based on wireless tags,

- $\quad$ several mobiles: traveling on a bus line, network, people at home, animals, etc.

- dominant mobiles: fleets, traffic, animals, etc.

- calculated behavior: systematic scanning,

- dynamic behavior: decisions programmed depending on the mobile history.

\section{REFERENCES}

[1] B. Pottier and P.-Y. Lucas, "Dynamic networks. NetGen : objectives, installation, use and programming," Universite' de Brest, https://github.com/NetGenProject/documentation/blob/master/DocNetGe n.pdf, Tech. Rep., February 2015.

[2] R. Dogaru and I. Dogaru., "An efficient sound propagation software simulator based on cellular automata." ISEEE - 3rd International Symposium, Sept. 2010.

[3] V. Terrier, "Two-dimensional cellular automata recognizer," Theor. Com-put.Sci., vol. 218(2), p. 325-346, 1999.

[4] S. A. Gelfand, Hearing: An Introduction to Psychological and Physiological Acoustics. informa Fifth Ed., 2010.

[5] Inmos, Occam 2.1 Reference Manual. SGS-THOMSON Microelectronics Limited, 1995.

[6] NVIDIA, NVIDIA CUDA Programming Guide 2.0. NVIDIA, 2008.

[7]

[8] T. Komatsuzaki and Y. Iwata., "Study on acoustic field with fractal boundary using cellular automata." Cellular Automata, vol. pp, pp. 282290, 2008.

[9] M. Sosnick and W. Hsu., "Implementing a finite difference-based realtime sound synthesizer using gpus." International Conference on New Interfaces for Musical Expression, 2011.

[10] T. M. E., "Partial differential equations - basic theory." Applied Mathematical Sciences, Springer-Verlag New York, vol. 115, 1996.

[11] M. Vacher and al., "Life sounds extraction and classification in noisy environment." SIP, 2003. 